Research Article

\title{
Numerical Approximation for Fractional Neutron Transport Equation
}

\author{
Zhengang Zhao $\mathbb{D}^{1}$ and Yunying Zheng $\mathbb{D}^{2}$ \\ ${ }^{1}$ Department of Fundamental Courses, Shanghai Customs College, Shanghai 201204, China \\ ${ }^{2}$ School of Mathematical Sciences, Huaibei Normal University, Huaibei 235000, China \\ Correspondence should be addressed to Yunying Zheng; zhengyunying1973@163.com
}

Received 21 December 2020; Revised 9 January 2021; Accepted 20 January 2021; Published 15 March 2021

Academic Editor: Imtiaz Ahmad

Copyright $\odot 2021$ Zhengang Zhao and Yunying Zheng. This is an open access article distributed under the Creative Commons Attribution License, which permits unrestricted use, distribution, and reproduction in any medium, provided the original work is properly cited.

\begin{abstract}
Fractional neutron transport equation reflects the anomalous transport processes in nuclear reactor. In this paper, we will construct the fully discrete methods for this type of fractional equation with Riesz derivative, where the generalized WENO5 scheme is used in spatial direction and Runge-Kutta schemes are adopted in temporal direction. The linear stabilities of the generalized WENO5 schemes with different stages and different order ERK are discussed detailed. Numerical examples show the combinations of forward Euler/two-stage, second-order ERK and WENO5 are unstable and the three-stage, third-order ERK method with generalized WENO5 is stable and can maintain sharp transitions for discontinuous problem, and its convergence reaches fifth order for smooth boundary condition.
\end{abstract}

\section{Introduction}

The fractional differential operations, such as the natural generalizations of the classical operators of integer order, have become more and more popular. Many phenomena in physics, engineering, biology, chemistry, and other sciences have been described successfully by fractional calculus and fractional differential equations [1]. Recently, several numerical methods to solve fractional differential equations have been developing rapidly. For example, the finite difference method [2-5], the finite element method [6-13], the discontinuous finite element method [14-20], the spectral method [21-24], the finite volume method $[25,26]$, etc. However, most of them are focusing on the fractional parabolic equation. It seems that there are few works regarding the numerical methods for fractional hyperbolic equation, where the physical phenomena often refer to vibration and oscillation of the complex material or complex progress. Compared to the classical hyperbolic equation, the solutions of fractional hyperbolic equation may also develop discontinuously even when the initial condition is smooth. It is impossible to deduce the analytical solutions in most cases, and one thus has to rely on numerical methods. So, it is very meaningful to study the numerical methods for fractional hyperbolic equation.

As a typical representative of hyperbolic equation, the classical neutron transport equation studies the motions and interactions of neutrons with materials in a nuclear reactor. Besides, the transport equation also plays an important role within the transport theory. Compared to the classical neutron transport equation, the fractional neutron transport equation denotes the anomalous transport processes [27], which was studied by many researchers. Das and Biswas [28] developed a constitutive neutron diffusion equation for describing the neutron flux profile by using the concept of fractional divergence. Vyawahare et al. [29] studied a fractional-order model of a nuclear reactor, which predicts subdiffusive behaviour for long time. Vyawahare and Nataraj [30] dealt with a fractional-order model of neutron transport process and pointed out that it is a more faithful and realistic representation of neutron movements than the classical model.

Weighted essentially nonoscillatory (WENO) schemes [31-34] are the effective numerical methods to solve the hyperbolic equation, specially, the fifth-order WENO 
(WENO5), which is a robust scheme for solving the classical neutron transport equation. Naturally, applying the WENO schemes to solve the fractional differential equation numerically is an interesting work. However, it seems that there are few works about the WENO schemes used to approximate the fractional differential equation except [3]. In [3], Deng et al. designed a WENO6 scheme for the fractional diffusion equation in the sense of Caputo derivative.
In this work, we will design the generalized WENO5 schemes to solve the following spatial fractional neutron transport equation:

$$
\frac{\partial u}{\partial t}+\lambda \frac{\partial^{\alpha} u}{\partial|x|^{\alpha}}=f(x, t)
$$

where $\lambda$ is a given constant coefficient and $\partial^{\alpha} u / \partial|x|^{\alpha}$ represents the Riesz derivative given by

$$
\frac{\partial^{\alpha} u}{\partial|x|^{\alpha}}=\frac{C_{\alpha}}{\Gamma(1-\alpha)} \frac{\mathrm{d}}{\mathrm{d} x}\left\{\int_{a}^{x}(x-\tau)^{-\alpha} u(\tau, t) \mathrm{d} \tau-\int_{x}^{b}(\tau-x)^{-\alpha} u(\tau, t) \mathrm{d} \tau\right\}
$$

in which $C_{\alpha}=-1 /(2 \cos ((\pi / 2) \alpha)), 0<\alpha<1$.

The basic idea of numerical methods in this paper is to split the $\alpha(0<\alpha<1)$ order fractional derivative into a fractional integral and a classical first derivative. Next, we apply the Gauss-Jacobi quadrature to approximate the fractional integral and WENO5 to approximate the classical first derivative separately. Then, the three-stage, third-order SSP $(3,3)$ explicit Runge-Kutta (ERK) method or the thirdorder TVD ERK method [35] is selected as the discrete time method to solve the ODE systems. The numerical experiments confirm fifth-order convergence order and show the maintenance of sharp discontinuous transitions. Furthermore, the linear stability of various ERK methods together with generalized WENO5 to approximate the fractional neutron transport equation is also discussed. From the linear stability analyses, we get that the combinations of the forward Euler scheme with WENO5 and any two-stage, second-order ERK schemes with WENO5 are linearly unstable in the fractional neutron transport equation. And, the combination of any three-stage, third-order ERK method with WENO5 for the fractional neutron transport equation is linearly stable. All of these theoretical results are demonstrated by numerical experiments.

The structure of this paper is as follows. In Section 2, the generalized WENO5 schemes of the spatial fractional neutron transport equation are designed, including the Gauss quadrature formula relative to Jacobi weights, to compute the fractional integral terms. Then, the linear stability analyses of the designed schemes are discussed in Section 3. Finally, the numerical examples are presented to verify the convergence and stability of the designed schemes in Section 4.

\section{The Generalized WENO5 Schemes}

To achieve our method, we need to introduce the fractional integrals and fractional derivatives.

Firstly, we define the $\alpha$ th order left- and right-fractional integrals of function $u(x)$ as follows:

$$
\begin{aligned}
& J_{a, x}^{\alpha} u(x)=\frac{1}{\Gamma(\alpha)} \int_{a}^{x} \frac{u(s)}{(x-s)^{1-\alpha}} \mathrm{d} s, \\
& J_{x, b}^{\alpha} u(x)=\frac{1}{\Gamma(\alpha)} \int_{x}^{b} \frac{u(s)}{(s-x)^{1-\alpha}} \mathrm{d} s,
\end{aligned}
$$

where $\alpha>0$. Next, we give the $\alpha$ th order left- and rightCaputo derivatives:

$$
\begin{aligned}
& { }_{C} D_{a, x}^{\alpha} u(x)=J_{a, x}^{n-\alpha}\left[u^{(n)}(x)\right], \\
& { }_{C} D_{x, b}^{\alpha} u(x)=(-1)^{n} J_{x, b}^{n-\alpha}\left[u^{(n)}(x)\right],
\end{aligned}
$$

in which $n-1<\alpha<n \in Z^{+}$.

According to [36], when $0<\alpha<1$, we can easily derive the following relations between two fractional derivatives:

$$
\frac{\partial^{\alpha} u}{\partial|x|^{\alpha}}=C_{\alpha}\left\{{ }_{C} D_{a, x}^{\alpha} u(x)+{ }_{C} D_{x, b}^{\alpha} u(x)+\frac{u(a)(x-a)^{-\alpha}-u(b)(b-x)^{-\alpha}}{\Gamma(1-\alpha)}\right\} .
$$

Therefore, equation (1) can be rewritten as

$$
\frac{\partial u(x, t)}{\partial t}+\lambda C_{\alpha}\left({ }_{C} D_{a, x}^{\alpha}+{ }_{C} D_{x, b}^{\alpha}\right) u(x, t)+\lambda C_{\alpha} \frac{u(a, t)(x-a)^{-\alpha}-u(b, t)(b-x)^{-\alpha}}{\Gamma(1-\alpha)}=f(x, t) .
$$


By using the definition of the Caputo derivative, we have

$$
\frac{\partial u(x, t)}{\partial t}+\lambda\left(J_{a, x}^{1-\alpha} v(x, t)-J_{x, b}^{1-\alpha} v(x, t)\right)+\lambda C_{\alpha} \frac{u(a, t)(x-a)^{-\alpha}-u(b, t)(b-x)^{-\alpha}}{\Gamma(1-\alpha)}=f(x, t),
$$

where $v(x, t)=(\partial / \partial x) u(x, t)$.

In order to get a high-order accuracy of the numerical solution, we apply Gauss-type quadrature formula relative to
Jacobi weights to compute the fractional integral terms in (7). Therefore, we transfer the integral intervals $[a, x]$ and $[x, b]$ to the standard interval $[-1,1]$, respectively:

$$
\begin{aligned}
& \int_{a}^{x}(x-\xi)^{-\alpha} v(\xi, t) \mathrm{d} \xi=\left(\frac{x-a}{2}\right)^{1-\alpha} \int_{-1}^{1}(1-\eta)^{-\alpha} v\left(\frac{a+x}{2}+\frac{x-a}{2} \eta, t\right) \mathrm{d} \eta \\
& \int_{x}^{b}(\xi-x)^{-\alpha} v(\xi, t) \mathrm{d} \xi=\left(\frac{b-x}{2}\right)^{1-\alpha} \int_{-1}^{1}(1+\eta)^{-\alpha} v\left(\frac{b+x}{2}+\frac{b-x}{2} \eta, t\right) \mathrm{d} \eta
\end{aligned}
$$

Now, we denote $h$ as the spatial step size, $h=(b-a) / N$, where $N$ is the numbers of subintervals divided in the spatial directions. Then, by using $N+1$ points Gauss-Jacobi quadrature and the Lagrange interpolation, the left- and right-fractional integrals in the right-hand side of (8) and (9) at the mesh points $x=x_{j}$ can be, respectively, approximated by

$$
\begin{gathered}
\int_{a}^{x_{j}}\left(x_{j}-\xi\right)^{-\alpha} v(\xi, t) \mathrm{d} \xi \sim\left(\frac{x_{j}-a}{2}\right)^{1-\alpha} \sum_{j=0}^{N} v\left(x_{j}, t\right)\left\{\sum_{k=0}^{N} F_{j}\left(\frac{a+x_{j}}{2}+\frac{x_{j}-a}{2} \eta_{k}\right) \omega_{k}\right\} \\
\int_{x_{j}}^{b}\left(\xi-x_{j}\right)^{-\alpha} v(\xi, t) \mathrm{d} \xi \sim\left(\frac{b-x_{j}}{2}\right)^{1-\alpha} \sum_{j=0}^{N} v\left(x_{j}, t\right)\left\{\sum_{k=0}^{N} F_{j}\left(\frac{b+x_{j}}{2}+\frac{b-x_{j}}{2} \eta_{k}\right) \widetilde{\omega}_{k}\right\},
\end{gathered}
$$

where $\left\{\eta_{k}\right\}_{k=0}^{N}$ is the Gauss nodes, $\left\{\omega_{k}\right\}_{k=0}^{N}$ and $\left\{\widetilde{\omega}_{k}\right\}_{k=0}^{N}$ are Jacobi weights, and $F_{j}$ is the Lagrange interpolation basis function associated with the points $\left\{x_{j}\right\}_{j=0}^{N}$, which can be replaced by any other kind of interpolation basis functions.
In this paper, we denote $u\left(x_{j}, t_{n}\right)$ and $u_{j}^{n}$ as the exact solution and the numerical solution at mesh point $\left(x_{j}, t_{n}\right)$, respectively. For the intermediate function $v\left(x_{j}, t\right)$, we use WENO5 to do the discretization as follows:

$$
v\left(x_{j}, t\right)=\frac{\partial u}{\partial x}\left(x_{j}, t\right)=\frac{1}{h}\left(\widehat{u}_{j+(1 / 2)}-\widehat{u}_{j-(1 / 2)}\right)+\mathcal{O}\left(h^{5}\right), \quad j=0,1, \ldots, N
$$

where the term $\widehat{u}_{j+(1 / 2)}$ is the numerical flux and can be split into positive and negative parts:

$$
\widehat{u}_{j+(1 / 2)}=\widehat{u}_{j+(1 / 2)}^{+}+\widehat{u}_{j+(1 / 2)}^{-} \text {. }
$$
$0,1,2$.

On stencil, $S_{r}(j)=\left\{I_{j-r}, \ldots, I_{j+s}\right\}, r+s+1=3, \quad r=$

For $\widehat{u}_{j+(1 / 2)}^{+}$and $\widehat{u}_{j+(1 / 2)}^{-}$, we define them on each stencil $S_{r}(j)$ as follows:

$$
\begin{aligned}
& \left.\widehat{u}_{j+(1 / 2)}^{+}=\omega_{0}^{+}\left(\frac{1}{3} u_{j-2}-\frac{7}{6} u_{j-1}+\frac{11}{6} u_{j}\right)+\omega_{1}^{+}\left(-\frac{1}{6} u_{j-1}+\frac{5}{6} u_{j}+\frac{1}{3} u_{j+1}\right)+\omega_{2}^{+}\left(\frac{1}{3} u_{j}+\frac{5}{6} u_{j+1}-\frac{1}{6} u_{j+2}\right)\right], \\
& \left.\widehat{u}_{j+(1 / 2)}^{-}=\omega_{0}^{-}\left(\frac{1}{3} u_{j+3}-\frac{7}{6} u_{j+2}+\frac{11}{6} u_{j+1}\right)+\omega_{1}^{-}\left(-\frac{1}{6} u_{j+2}+\frac{5}{6} u_{j+1}+\frac{1}{3} u_{j}\right)+\omega_{2}^{-}\left(\frac{1}{3} u_{j+1}+\frac{5}{6} u_{1}-\frac{1}{6} u_{j-1}\right)\right],
\end{aligned}
$$


where, for parameters $\omega_{k}^{ \pm}$, stencil $S_{r}(j)$, and the other details of the WENO5 method, one can refer to [31, 34, 37].

Next, we will use the forward Euler method, SSP $(3,3)$ schemes, and some other Runge-Kutta schemes to solve the ODE system in the temporal direction after doing the spatial discretization.

\section{Linear Stability Analysis}

In this section, we will discuss the linear stability properties of the generalized WENO5 method when coupled with different kinds of ERK methods for solving the fractional neutron transport equation (1). Here, we define CFL number as $\sigma=\lambda \Delta t / h$ and only consider the corresponding homogeneous problem:

$$
\frac{\partial u(x, t)}{\partial t}+\frac{\partial^{\alpha} u}{\partial|x|^{\alpha}}(x, t)=0,
$$

under homogeneous boundary conditions $u(a, t)=u(b$, $t)=0$.

In the following, we will show that the combination of the generalized WENO5 and forward Euler scheme for the fractional neutron transport equation (15) is linearly unstable.
Lemma 1 (see [37]). When the forward Euler method is applied with the classical WENO5 scheme, the function $\left(\widehat{u}_{j+(1 / 2)}^{+, n}-\widehat{u}_{j-(1 / 2)}^{+, n}\right) / u_{j}^{n}$, which is defined as $z(\phi)$, can be approximated by

$$
z(\phi)=\frac{7}{60} \phi^{6}+\mathcal{O}\left(\phi^{8}\right)+\imath\left(\phi+\mathcal{O}\left(\phi^{7}\right)\right)
$$

where the Taylor expansions of $\sin \phi$ and $\cos \phi$ are used, $\phi$ is a small positive number, only related to the spatial step $h$, and 1 is the imaginary unit, i.e., $\imath^{2}=-1$.

Theorem 1. The combination of the generalized WENO5 method and the forward Euler method for the fractional neutron transport equation (15) is linearly unstable.

Proof. Here, we only consider the Lax-Frichrichs flux splitting, which means that the negative part of the numerical flux is zero [37]. And, the same analysis and conclusions can be shifted to the other flux splitting.

According to the Fourier transform, we assume

$$
u_{j}=\widehat{u} e^{i j \phi},
$$

where $\phi=\xi h$, and $\xi$ is the spatial frequency. By using (13) and (17), we further conclude that

$$
\widehat{u}_{j+(1 / 2)}^{+}=u_{j}\left[\omega_{0}^{+}\left(\frac{1}{3} e^{-2 \imath \phi}-\frac{7}{6} e^{-\imath \phi}+\frac{11}{6}\right)+\omega_{1}^{+}\left(-\frac{1}{6} e^{-\imath \phi}+\frac{5}{6}+\frac{1}{3} e^{\imath \phi}\right)+\omega_{2}^{+}\left(\frac{1}{3}+\frac{5}{6} e^{\imath \phi}-\frac{1}{6} e^{2 \imath \phi}\right)\right] .
$$

When the forward Euler method is adopted, the fully discrete generalized WENO5 method becomes

$$
u_{j}^{n+1}=u_{j}^{n}-\sigma \frac{C_{\alpha}}{\Gamma(1-\alpha)} \sum_{j=0}^{N} L\left(u_{j-3}^{n}, u_{j-2}^{n}, \ldots, u_{j+2}^{n}\right)\left(\left(\frac{x_{j}-a}{2}\right)^{1-\alpha} \sum_{k=0}^{N} F_{j}\left(\frac{a+x_{j}}{2}+\frac{x_{j}-a}{2} \eta_{k}\right) \omega_{k}-\left(\frac{b-x_{j}}{2}\right)^{1-\alpha} \sum_{k=0}^{N} F_{j}\left(\frac{b+x_{j}}{2}+\frac{b-x_{j}}{2} \eta_{k}\right) \widetilde{\omega}_{k}\right),
$$

where $L\left(u_{j-3}^{n}, u_{j-2}^{n}, \ldots, u_{j+2}^{n}\right)=\widehat{u}_{j+(1 / 2)}^{+, n}-\widehat{u}_{j-(1 / 2)}^{+, n}$, which can

Then, equation (15) becomes be defined as $z(\phi) u_{j}^{n}$.

$$
\begin{aligned}
u_{j}^{n+1} & =u_{j}^{n}-\sigma \frac{C_{\alpha}}{\Gamma(1-\alpha)} \sum_{j=0}^{N} z(\phi) u_{j}^{n}\left[\left(\frac{x_{j}-a}{2}\right)^{1-\alpha} \sum_{k=0}^{N} F_{j}\left(\frac{a+x_{j}}{2}+\frac{x_{j}-a}{2} \eta_{k}\right) \omega_{k}-\left(\frac{b-x_{j}}{2}\right)^{1-\alpha} \sum_{k=0}^{N} F_{j}\left(\frac{b+x_{j}}{2}+\frac{b-x_{j}}{2} \eta_{k}\right) \widetilde{\omega}_{k}\right] \\
& =u_{j}^{n}\left\{1-\sigma \frac{C_{\alpha}}{\Gamma(1-\alpha)} \sum_{j=0}^{N} z(\phi)\left[\left(\frac{x_{j}-a}{2}\right)^{1-\alpha} \sum_{k=0}^{N} F_{j}\left(\frac{a+x_{j}}{2}+\frac{x_{j}-a}{2} \eta_{k}\right) \omega_{k}-\left(\frac{b-x_{j}}{2}\right)^{1-\alpha} \sum_{k=0}^{N} F_{j}\left(\frac{b+x_{j}}{2}+\frac{b-x_{j}}{2} \eta_{k}\right) \widetilde{\omega}_{k}\right]\right\} .
\end{aligned}
$$


Define the amplification factor:

$$
g(\sigma z)=1-\sigma \frac{C_{\alpha}}{\Gamma(1-\alpha)} \sum_{j=0}^{N} z(\phi)\left[\left(\frac{x_{j}-a}{2}\right)^{1-\alpha} \sum_{k=0}^{N} F_{j}\left(\frac{a+x_{j}}{2}+\frac{x_{j}-a}{2} \eta_{k}\right) \omega_{k}-\left(\frac{b-x_{j}}{2}\right)^{1-\alpha} \sum_{k=0}^{N} F_{j}\left(\frac{b+x_{j}}{2}+\frac{b-x_{j}}{2} \eta_{k}\right) \widetilde{\omega}_{k}\right] .
$$

By Lemma 1, we get the amplification factor as follows:

$$
\begin{aligned}
g(\sigma z) & =1-\sigma \frac{C_{\alpha}}{\Gamma(1-\alpha)}\left(\mathcal{O}\left(\phi^{6}\right)+\iota\left(\phi+\mathcal{O}\left(\phi^{7}\right)\right) \sum_{j=0}^{N}\left[\left(\frac{x_{j}-a}{2}\right)^{1-\alpha} \sum_{k=0}^{N} F_{j}\left(\frac{a+x_{j}}{2}+\frac{x_{j}-a}{2} \eta_{k}\right) \omega_{k}-\left(\frac{b-x_{j}}{2}\right)^{1-\alpha} \sum_{k=0}^{N} F_{j}\left(\frac{b+x_{j}}{2}+\frac{b-x_{j}}{2} \eta_{k}\right) \widetilde{\omega}_{k}\right]\right. \\
& =1-\sigma\left(\mathcal{O}\left(\phi^{6}\right)+\iota\left(\phi+\mathcal{O}\left(\phi^{7}\right)\right) \sum_{j=0}^{N} \sum_{k=0}^{N}\left[c_{j} F_{j}\left(\frac{a+x_{j}}{2}+\frac{x_{j}-a}{2} \eta_{k}\right) \omega_{k}+d_{j} F_{j}\left(\frac{b+x_{j}}{2}+\frac{b-x_{j}}{2} \eta_{k}\right) \tilde{\omega}_{k}\right],\right.
\end{aligned}
$$

where $\quad\left(C_{\alpha} / \Gamma(1-\alpha)\right)\left(\left(x_{j}-a\right) / 2\right)^{1-\alpha} \quad$ and $-\left(C_{\alpha} / \Gamma(1-\alpha)\right)\left(\left(b-x_{j}\right) / 2\right)^{1-\alpha}$ are denoted by $c_{j}$ and $d_{j}$ According to a simple calculation, we can get that correspondingly.

$$
|g|^{2}=1+\sigma^{2}\left[\sum_{j=0}^{N} \sum_{k=0}^{N}\left(c_{j} F_{j}\left(\frac{a+x_{j}}{2}+\frac{x_{j}-a}{2} \eta_{k}\right) \omega_{k}+d_{j} F_{j}\left(\frac{b+x_{j}}{2}+\frac{b-x_{j}}{2} \eta_{k}\right) \widetilde{\omega}_{k}\right)\right]^{2} \phi^{2}+\mathcal{O}\left(\phi^{6}\right)
$$

$>1, \quad \forall \sigma>0$

The proof is complete.

Next, we consider the following $s$-stage ERK method to replace the forward Euler method:

$$
\begin{aligned}
y_{n}^{1} & =y_{n}, \\
y_{n}^{k} & =y_{n}+\Delta t \sum_{i=1}^{k-1} a_{k, i} f\left(t_{n}+c_{i} \Delta t, y_{n}^{i}\right), \quad k=2, \ldots, s, \\
y_{n+1} & =y_{n}+\Delta t \sum_{i=1}^{s} b_{i} f\left(t_{n}+c_{i} \Delta t, y_{n}^{i}\right),
\end{aligned}
$$

where the coefficients satisfy $c_{k}=\sum_{j=1}^{k-1} a_{k, j}$, for $k=1, \ldots, s$, and $\sum_{i=1}^{s} b_{i}=1$.
Lemma 2 (see $[38,39]$ ). The amplification factor of the s-stage, p-order ERK method is

$g(\widehat{z})=1+\sum_{l=1}^{p} \frac{1}{l !} \widehat{z}^{l}+\sum_{l=p+1}^{s}\left(\sum_{j=l}^{s} b_{j}\left(\sum_{j>m_{1}>\cdots>m_{l-1} \leq 1} a_{j, m_{1}} a_{m_{1}, m_{2}} \cdots a_{m_{l-2}, m_{l-1}}\right)\right) \hat{z}^{l}=1+\sum_{l=1}^{p} \frac{1}{l} \widehat{z}^{l}+\sum_{l=p+1}^{s} \hat{z}^{l} b^{T} A^{l-1} e$,

where $b=\left(b_{1}, b_{2}, \ldots, b_{s}\right), A=\left(a_{i j}\right)_{1 \leq i, j \leq s}$ are the coefficients of the s-stage, $p$-order ERK method, $e=(1,1, \ldots, 1)$, and $\widehat{z}=-\sigma z$, where $z$ is caused by the character of the spatial operator. 
Remark that, from Theorem 1, we can see that the operator $\hat{z}$ should be adjusted to $-\sigma \sum_{j=0}^{N} z(\phi)$ $\sum_{k=0}^{N}\left(c_{j} F_{j}\left(\left(a+x_{j} / 2\right)+\left(x_{j}-a / 2\right) \eta_{k}\right) \omega_{k}+d_{j} F_{j}\left(\left(b+x_{j} / 2\right)+\right.\right.$ $\left.\left.\left(b-x_{j} / 2\right) \eta_{k}\right) \quad \widetilde{\omega}_{k}\right)$ in the fractional neutron transport equation, which is caused by the left and right spatial fractional integral terms.
Theorem 2. The combination of the generalized WENO5 method with any two-stage, second-order ERK method for the fractional neutron transport equation (15) is linearly unstable.

Proof. According to Lemmas 1 and 2, the amplification factor can be written as

$$
\begin{aligned}
g(\widehat{z})= & 1+\widehat{z}+\frac{1}{2} \widehat{z}^{2} \\
= & 1-\sigma \sum_{j=0}^{N} z(\phi) \sum_{k=0}^{N}\left[c_{j} F_{j}\left(\frac{a+x_{j}}{2}+\frac{x_{j}-a}{2} \eta_{k}\right) \omega_{k}+d_{j} F_{j}\left(\frac{b+x_{j}}{2}+\frac{b-x_{j}}{2} \eta_{k}\right) \widetilde{\omega}_{k}\right] \\
& +\frac{1}{2} \sigma^{2}\left[\sum_{j=0}^{N} z(\phi) \sum_{k=0}^{N}\left(c_{j} F_{j}\left(\frac{a+x_{j}}{2}+\frac{x_{j}-a}{2} \eta_{k}\right) \omega_{k}+d_{j} F_{j}\left(\frac{b+x_{j}}{2}+\frac{b-x_{j}}{2} \eta_{k}\right) \widetilde{\omega}_{k}\right)\right]^{2} \\
= & 1-\sigma\left[\frac{7}{60} \phi^{6}+\mathcal{O}\left(\phi^{8}\right)+1\left(\phi+\mathcal{O}\left(\phi^{7}\right)\right)\right]\left[\sum_{k=0}^{N} \sum_{j=0}^{N} c_{j} F_{j}\left(\frac{a+x_{j}}{2}+\frac{x_{j}-a}{2} \eta_{k}\right) \omega_{k}+d_{j} F_{j}\left(\frac{b+x_{j}}{2}+\frac{b-x_{j}}{2} \eta_{k}\right) \widetilde{\omega}_{k}\right] \\
& +\frac{1}{2} \sigma^{2}\left[\frac{7}{60} \phi^{6}+\mathcal{O}\left(\phi^{8}\right)+1\left(\phi+\mathcal{O}\left(\phi^{7}\right)\right)\right]^{2}\left[\sum_{k=0}^{N} \sum_{j=0}^{N} c_{j} F_{j}\left(\frac{a+x_{j}}{2}+\frac{x_{j}-a}{2} \eta_{k}\right) \omega_{k}+d_{j} F_{j}\left(\frac{b+x_{j}}{2}+\frac{b-x_{j}}{2} \eta_{k}\right) \widetilde{\omega}_{k}\right]^{2} \\
= & 1-\kappa \sigma\left[\frac{7}{60} \phi^{6}+\mathcal{O}\left(\phi^{8}\right)+1\left(\phi+\mathcal{O}\left(\phi^{7}\right)\right)\right]+\frac{1}{2} \kappa^{2} \sigma^{2} \\
& +\left[\frac{7}{60} \phi^{6}+\mathcal{O}\left(\phi^{8}\right)+1\left(\phi+\mathcal{O}\left(\phi^{7}\right)\right)\right]^{2},
\end{aligned}
$$

where the constant $\sum_{k=0}^{N} \sum_{j=0}^{N}\left[c_{j} F_{j}\left(\left(a+x_{j} / 2\right)+\left(x_{j}-\right.\right.\right.$ $\left.\left.a / 2) \eta_{k}\right) \omega_{k}+d_{j} F_{j}\left(\left(b+x_{j} / 2\right)+\left(b-x_{j} / 2\right) \eta_{k}\right) \widetilde{\omega}_{k}\right]$ is denoted by $\kappa$.

Then, we have that

$$
\begin{aligned}
|g|^{2} & =\left(1-\frac{1}{2} \kappa^{2} \sigma^{2} \phi^{2}+\mathcal{O}\left(\phi^{6}\right)\right)^{2}+\left(-\kappa \sigma \phi+\mathcal{O}\left(\phi^{7}\right)\right)^{2} \\
& =1+\frac{1}{4} \kappa^{4} \sigma^{4} \phi^{4}+\mathcal{O}\left(\phi^{6}\right) \\
& >1, \quad \forall \sigma>0 .
\end{aligned}
$$

The proof is finished.

Theorem 3. The combination of the generalized WENO5 method with any three-stage, three-order ERK method for the fractional neutron transport equation (15) is linearly stable.

Proof. According to Lemmas 1 and 2 and the proof of Theorem 2, we can get the amplification factor as follows:

$$
\begin{aligned}
g(\widehat{z})= & 1+\widehat{z}+\frac{1}{2} \widehat{z}^{2}+\frac{1}{6} \widehat{z}^{3} \\
= & 1-\kappa \sigma\left[\frac{7}{60} \phi^{6}+\mathcal{O}\left(\phi^{8}\right)+\imath\left(\phi+\mathcal{O}\left(\phi^{7}\right)\right)\right] \\
& +\frac{1}{2} \kappa^{2} \sigma^{2}\left[\frac{7}{60} \phi^{6}+\mathcal{O}\left(\phi^{8}\right)+\imath\left(\phi+\mathcal{O}\left(\phi^{7}\right)\right)\right]^{2} \\
& -\frac{1}{6} \kappa^{3} \sigma^{3}\left[\frac{7}{60} \phi^{6}+\mathcal{O}\left(\phi^{8}\right)+\imath\left(\phi+\mathcal{O}\left(\phi^{7}\right)\right)\right]^{3} .
\end{aligned}
$$

Then, we have that

$$
|g|^{2}=\left(1-\frac{1}{2} \kappa^{2} \sigma^{2} \phi^{2}-\frac{7}{60} \kappa \sigma \phi^{6}+\mathcal{O}\left(\phi^{8}\right)\right)^{2}
$$

$$
\begin{aligned}
& +\left(-\kappa \sigma \phi+\frac{1}{6} \kappa^{3} \sigma^{3} \phi^{3}+\mathcal{O}\left(\phi^{7}\right)\right)^{2} \\
= & 1-\frac{1}{12} \kappa^{4} \sigma^{4} \phi^{4}+\left(-\frac{7}{60} \kappa \sigma+\frac{1}{36} \kappa^{6} \sigma^{6}\right) \phi^{6}+\left(\mathcal{O}\left(\phi^{8}\right)\right)^{2}
\end{aligned}
$$$$
<1, \quad \forall \sigma>0 \text {. }
$$

The proof is finished. 
From the above proof, we can see that the conclusion of the linear stabilities for the combination of the generalized WENO5 method with the ERK method in the fractional neutron transport equation is the same as in the classical neutron transport equation. The reason is that the added fractional integral terms are approximated by a linear combination, in which the weights are bounded in the Gauss-Jacobi quadrature formulas. Therefore, all of the conclusions about the linear stabilities for the classical hyperbolic conservation law [37] can be shifted to problem (1), where the proofs are omitted.

\section{Numerical Examples and Results}

In this section, we will design three numerical examples to examine the effectiveness of the numerical algorithms. The first example with periodic boundary conditions is designed to verify the linear stability of the proposed schemes. The second example with two points' boundary conditions is computed to verify the convergence order. And, the third example is designed for numerical solving piecewise smooth solution and discontinuous initial condition.

Example 1. Consider the following fractional neutron transport equation:

$$
\frac{\partial u(x, t)}{\partial t}+{ }_{R L} D_{0, x}^{\alpha} u(x, t)=f(x, t), \quad x \in[0,1],
$$

where $u(x, t)=e^{-t}[\sin (4 \pi x)-2 \sin (2 \pi x)]$ is chosen as the exact solution.

Firstly, we use the forward Euler method with the WENO5 scheme. Next, we use the two-stage, second-order SSP ERK method with the WENO5 scheme. We compute the two cases for $\alpha=0.9$ and $\sigma=0.3,0.5,0.8,1.1$, which are shown in Figures 1 and 2 separately. According to the theoretical analyses, spurious oscillations due to linear instabilities are present.

Then, we use the SSP $(3,3)$ ERK method with the WENO5 scheme. $L^{\infty}$ error and convergence order are shown in Table 1. From Table 1, we can see that the numerical scheme performs well and shows the fifth-order convergence accuracy.

Example 2. Consider the following fractional neutron transport equation:

$$
\frac{\partial u(x, t)}{\partial t}+{ }_{R L} D_{0, x}^{\alpha} u(x, t)=f(x, t), \quad x \in[0,1],
$$

with the initial condition, two points' boundary conditions,

$$
\begin{aligned}
& u(x, 0)=x^{4}(1-x)^{3}, \\
& u(0, t)=u(1, t)=0,
\end{aligned}
$$

and the source term is

$$
f(x, t)=e^{-t}\left(-x^{4}+3 x^{5}-3 x^{5}+x^{7}+a x^{4-\alpha}+b x^{5-\alpha}+c x^{6-\alpha}+d x^{7-\alpha}\right)
$$

where $a=-24 / \Gamma(5-\alpha), b=360 / \Gamma(6-\alpha), c=-2160 / \Gamma(7-$ $\alpha)$, and $d=5040 / \Gamma(8-\alpha)$. Then, the exact solution is $u(x, t)=e^{-t} x^{4}(1-x)^{3}$.

Applying the SSP $(3,3)$ method with the WENO5 scheme, we get $L^{\infty}$ error and convergence order in Table 2. And the fifth-order convergence of the combination of the SSP $(3,3)$ method with the WENO5 scheme is still observed.

Example 3. Suppose that $[a, b]=[-10,10]$. Consider the fractional neutron transport equation as follows:

$$
\frac{\partial u(x, t)}{\partial t}+\frac{\partial^{\alpha} u}{\partial|x|^{\alpha}}(x, t)=0, \quad x \in[-10,10]
$$

with the boundary conditions $u(-10, t)=1, u(10, t)=0$, and the initial condition

$$
u(x, 0)= \begin{cases}1, & x \leq 0 \\ 0, & x>0\end{cases}
$$

In the simulation of Figure 3, we apply the SSP $(3,3)$ ERK method with the WENO5 scheme and take $N=200$ and $\sigma=0.1$. Figure 3 shows numerical solutions with different orders of fractional derivative, $\alpha=0.2,0.4,0.6,0.8$ at $T=0.1$. It can be seen that sharp transitions are retained in the numerical simulations for different $\alpha$.

Figure 4 shows the numerical solutions and their absolute errors for $\alpha=0.9, N=100$ and 200, and $\sigma=0.1$ at $T=1$. It can be seen that the bigger the $N$ value, the sharper the transitions in the simulations.

Figure 5 shows the numerical solutions when the parameters are chosen as $T=0.1, \alpha=0.9, N=200$, and $\sigma=$ $0.1,0.3,0.5,0.7$ separately. 


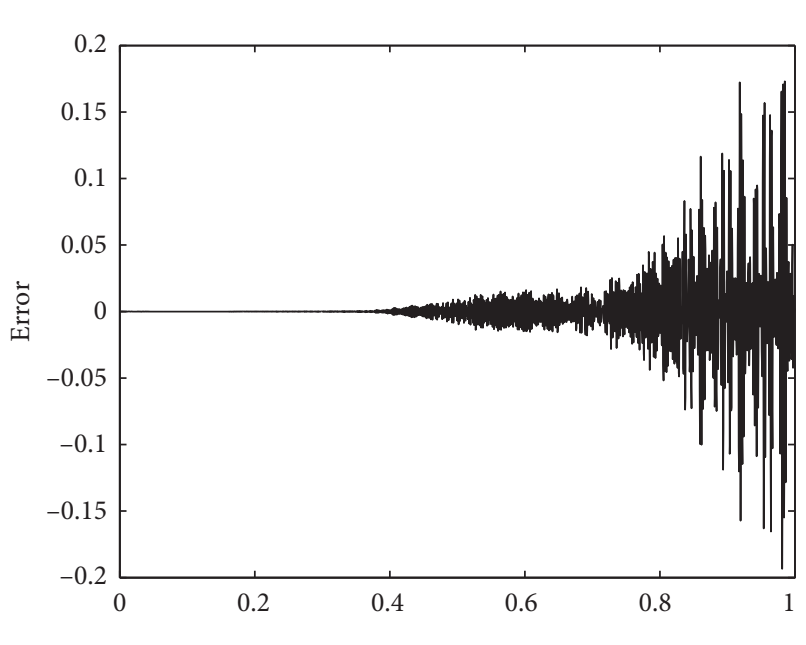

Absolute error

(a)

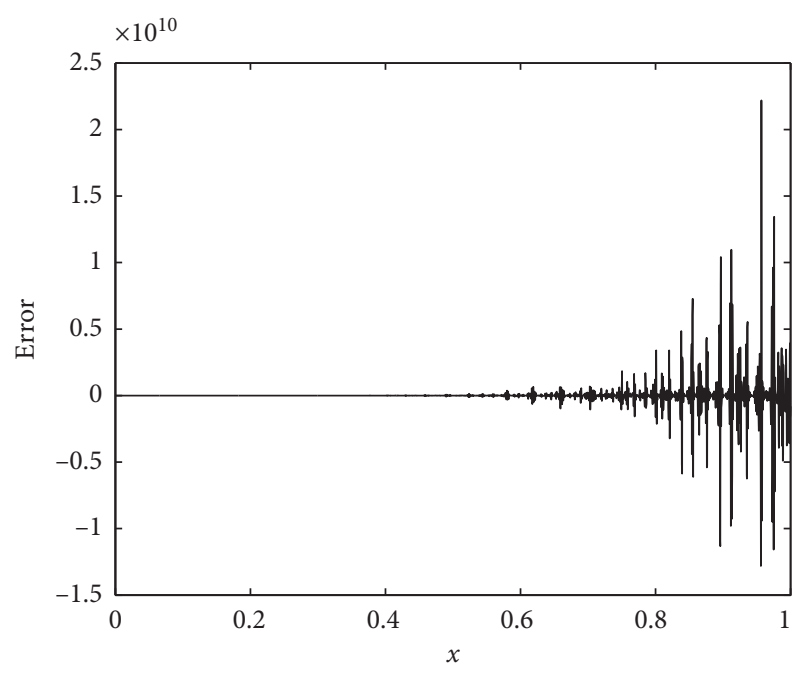

(c)

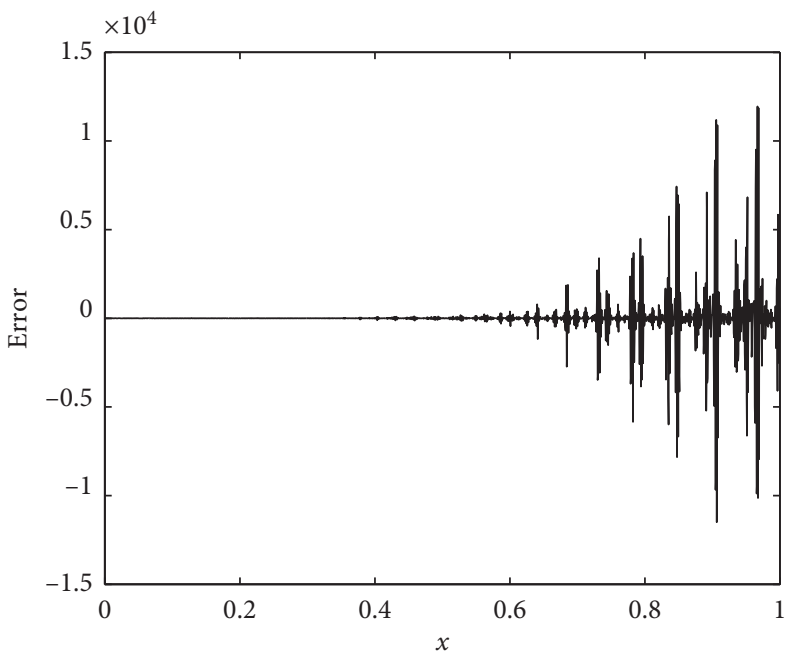

— Absolute error

(b)

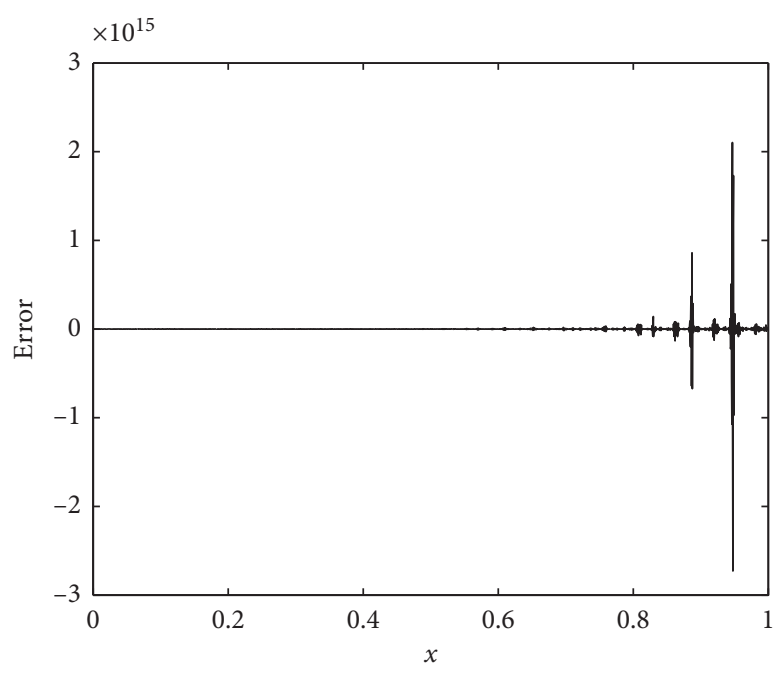

(d)

Figure 1: Absolute error of Example 1 with the forward Euler method and WENO5 scheme for different CFL numbers at $T=0.1, N=2560$, and $\alpha=0.9$. (a) $\sigma=0.3$, (b) $\sigma=0.5$, (c) $\sigma=0.8$, and (d) $\sigma=1.1$. 


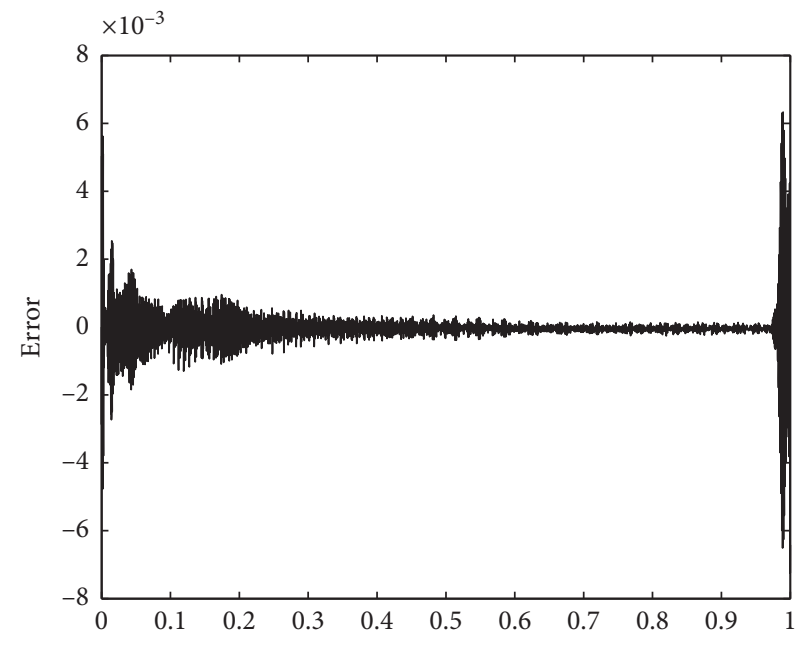

- Absolute error

(a)

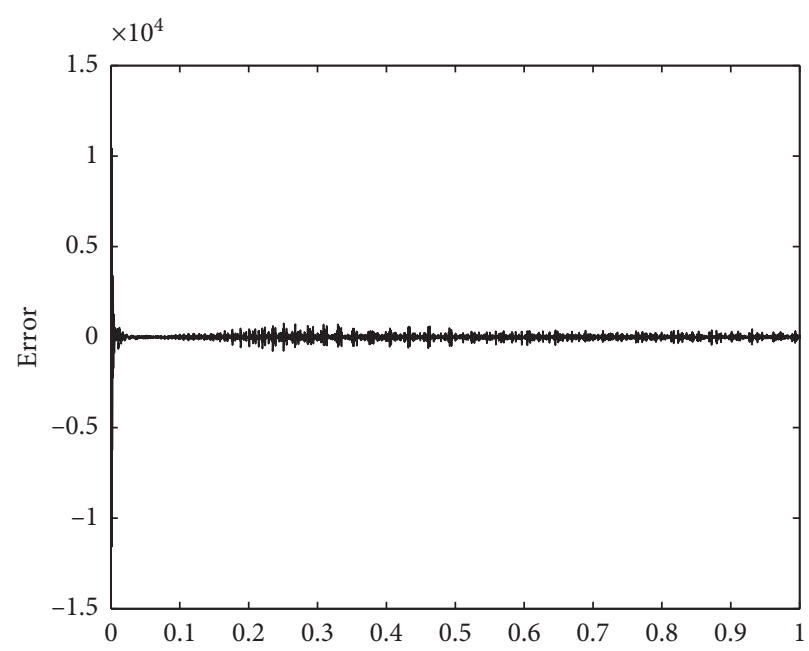

(c)

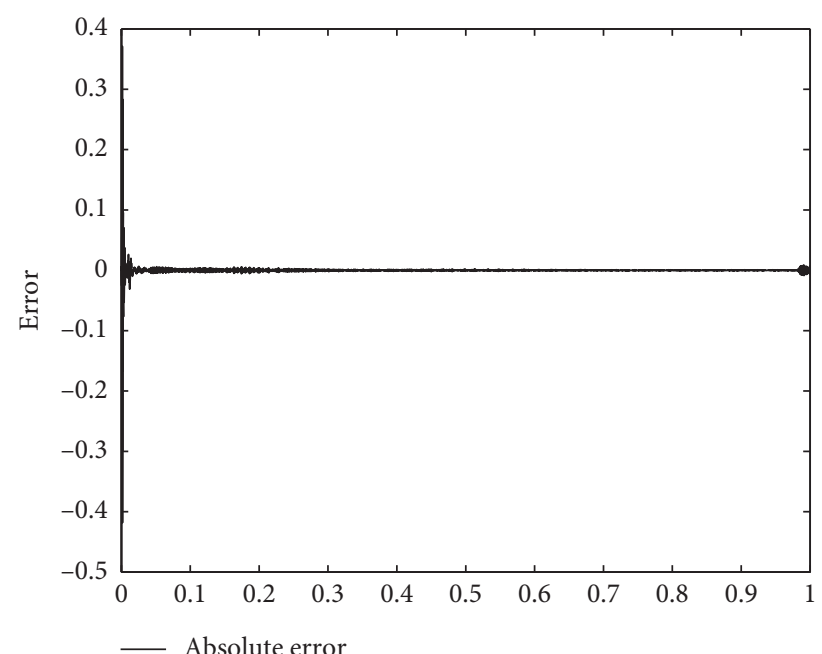

(b)

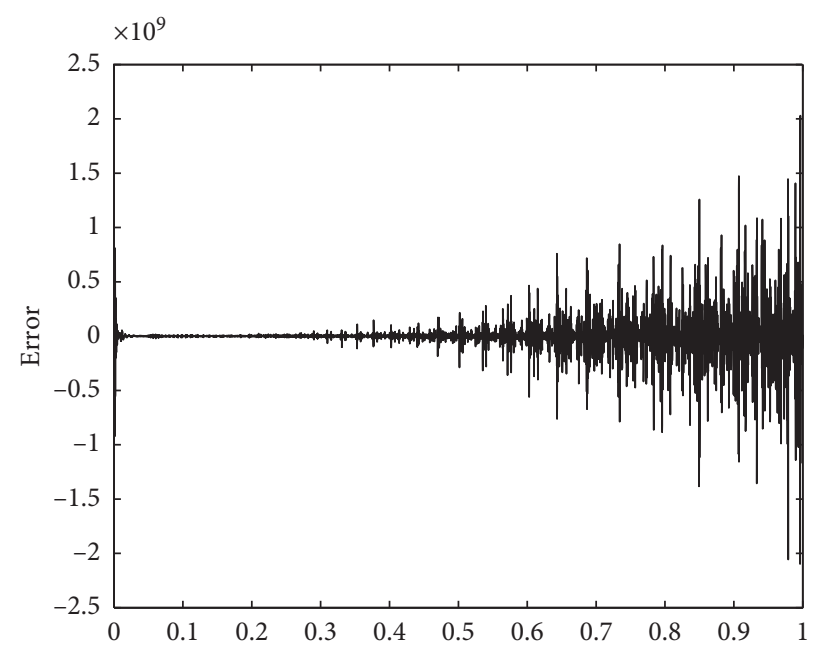

(d)

FIgURe 2: Absolute error of Example 1 with the two-stage, second-order ERK method and WENO5 scheme for different CFL numbers at $T=0.1, N=10240$, and $\alpha=0.9$. (a) $\sigma=0.3$, (b) $\sigma=0.5$, (c) $\sigma=0.8$, and (d) $\sigma=1.1$.

TABLE 1: Errors and convergence rates of Example 1 with the thirdorder TVD ERK method and WENO5 scheme for $\alpha=0.9$ and $\sigma=$ 0.4 at $T=0.1$.

\begin{tabular}{lcc}
\hline$N$ & $L^{\infty}$ & cv.rate \\
\hline 40 & $4.6260 e-003$ & - \\
80 & $1.0090 e-003$ & 2.1968 \\
160 & $1.8011 e-005$ & 5.8079 \\
320 & $3.4944 e-007$ & 5.6877 \\
\hline
\end{tabular}

TABLE 2: The $L^{\infty}$ errors and convergence rates of Example 2 with the third-order TVD ERK method and WENO5 scheme for $\alpha=0.9$ and $\sigma=0.4$ at $T=0.1$.

\begin{tabular}{lcc}
\hline$N$ & $L^{\infty}$ & cv.rate \\
\hline 40 & $2.3533 e-006$ & - \\
80 & $1.0556 e-007$ & 4.4786 \\
160 & $7.5624 e-009$ & 3.8031 \\
320 & $2.6494 e-010$ & 4.8351 \\
\hline
\end{tabular}




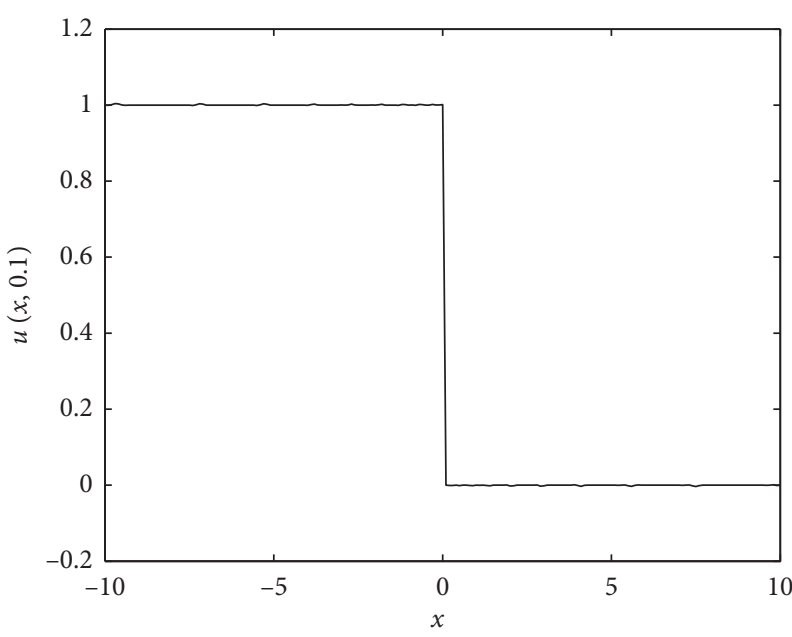

$-\alpha=0.2$

(a)

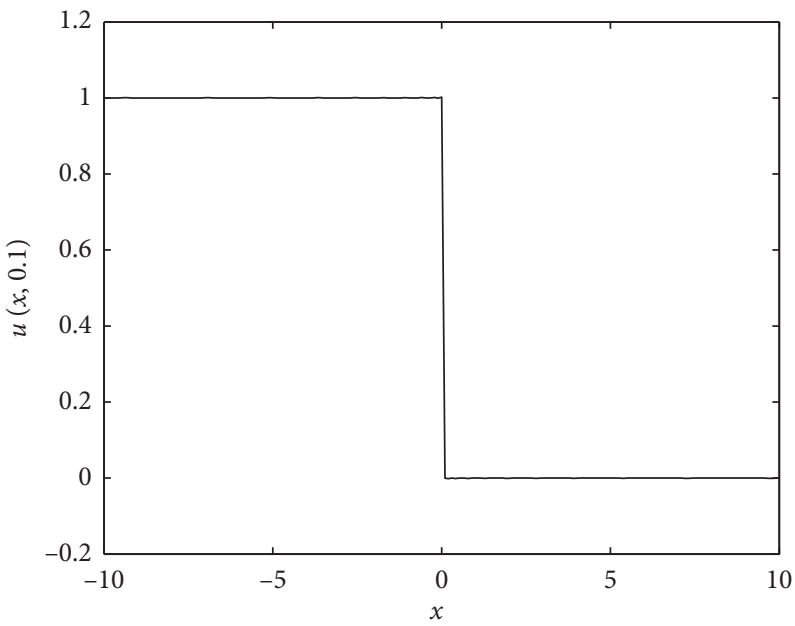

$-\alpha=0.6$

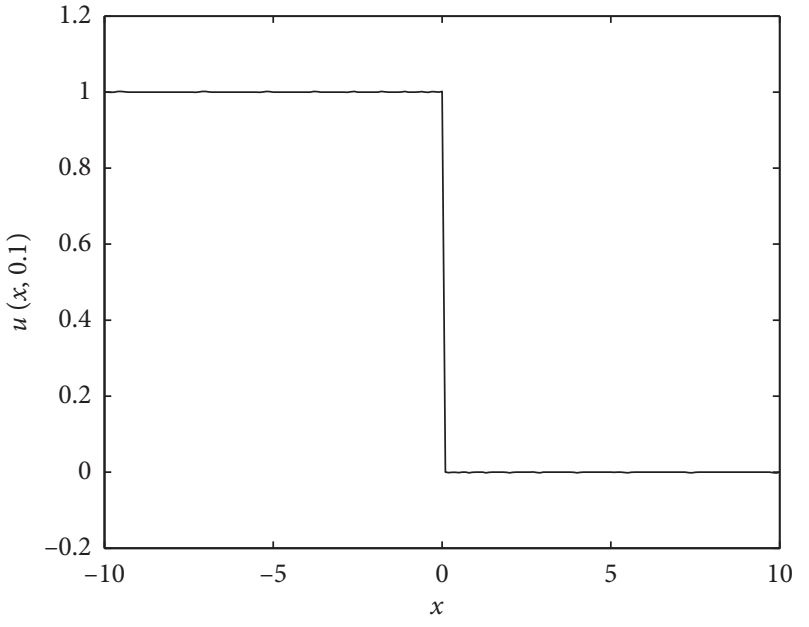

$-\alpha=0.4$

(b)

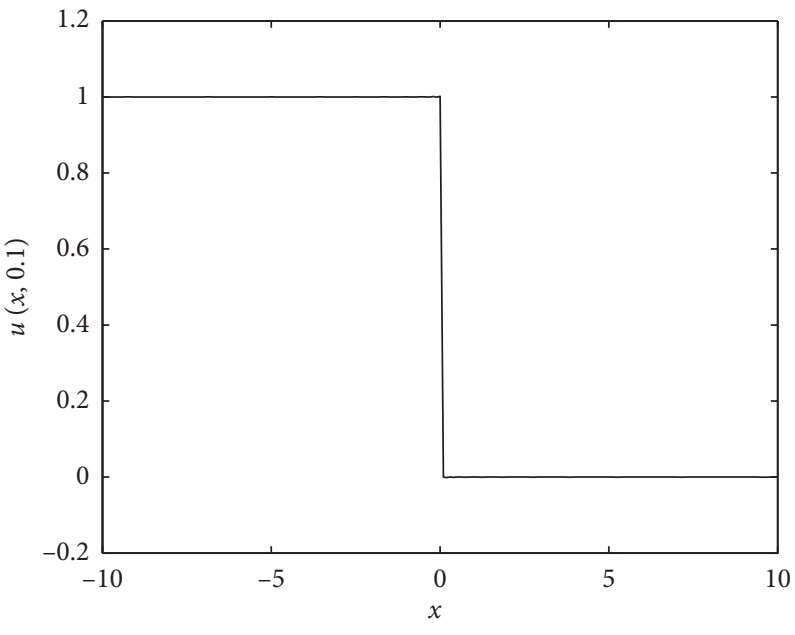

$-\alpha=0.8$

(c)

(d)

Figure 3: Numerical solutions of Example 3 with $N=200$ and $\sigma=0.1$ for different orders of fractional derivatives at $T=0.1$. (a) $\alpha=0.2$, (b) $\alpha=0.4$, (c) $\alpha=0.6$, and (d) $\alpha=0.8$. 


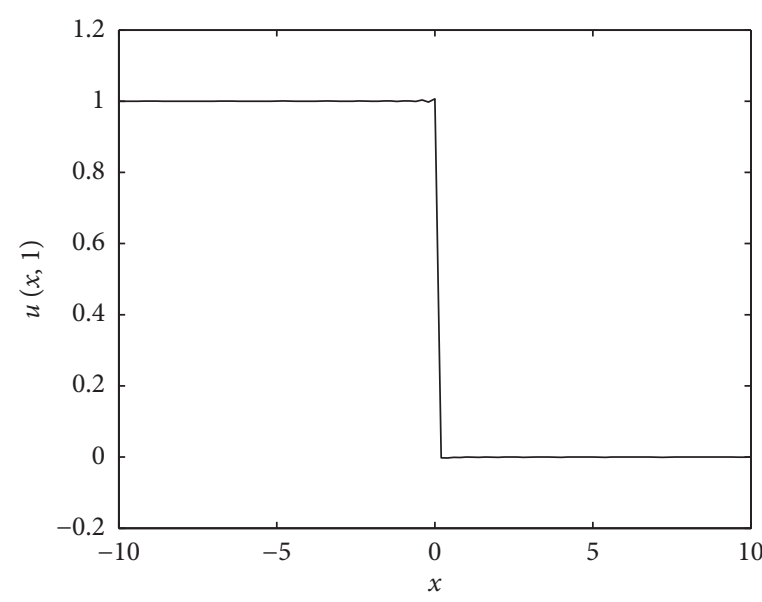

$-N=100$

(a)

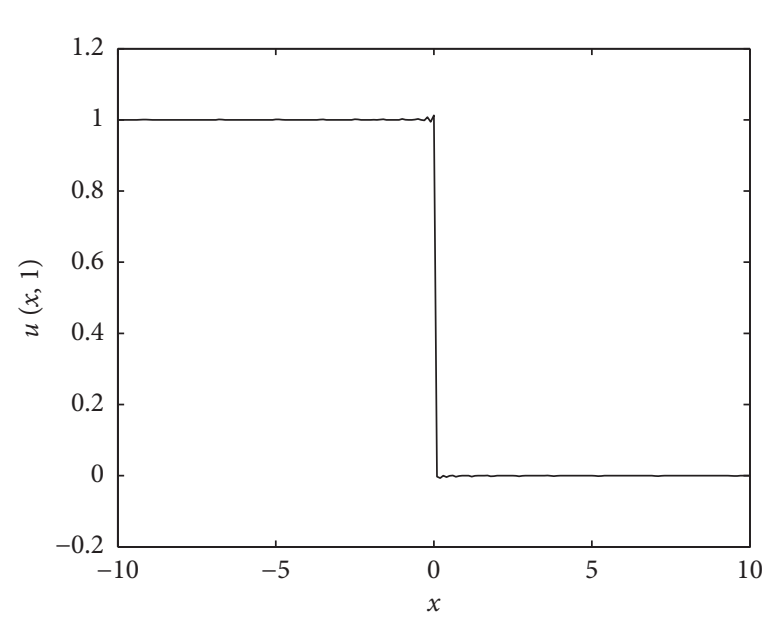

$-N=200$

(c)

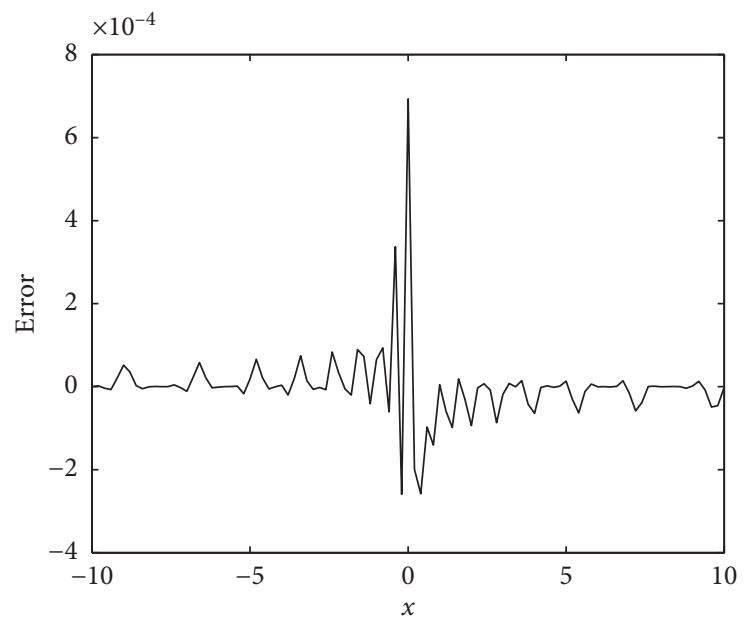

Absolute error

(b)

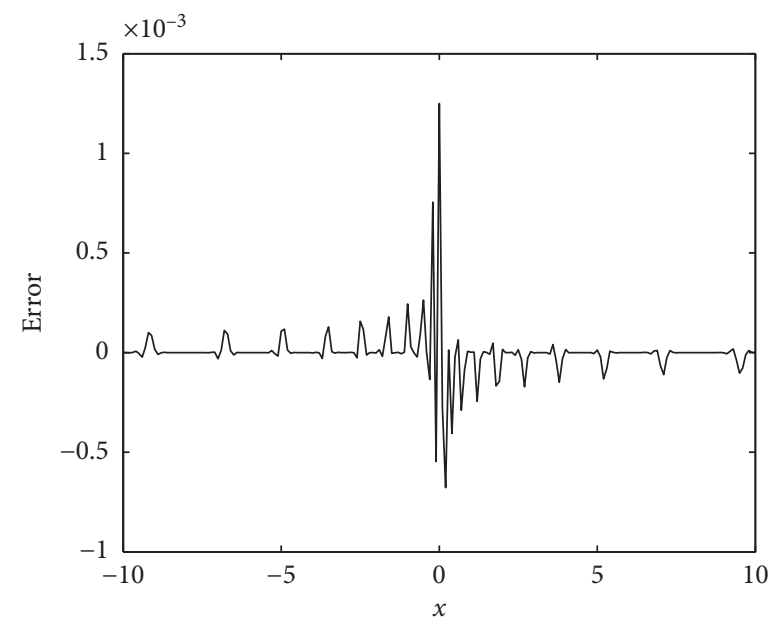

— Absolute error

(d)

Figure 4: The numerical solutions and their absolute errors of Example 3 with $\alpha=0.9$ and $\sigma=0.1$ for different $N$ at $T=1$. (a) $N=100$, (b) $N=100$, (c) $N=200$, and (d) $N=200$. 


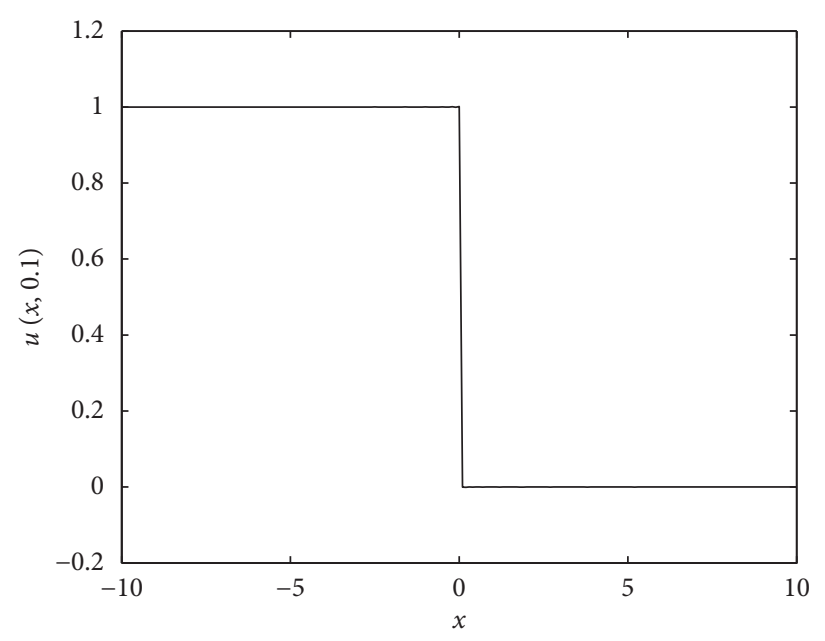

$-\Delta t=0.1 \mathrm{~h}$

(a)

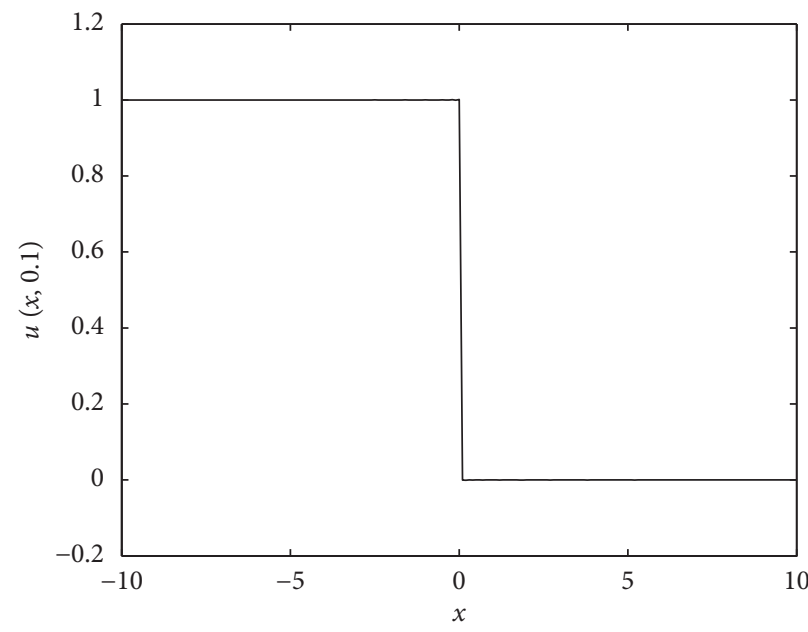

$-\Delta t=0.5 \mathrm{~h}$

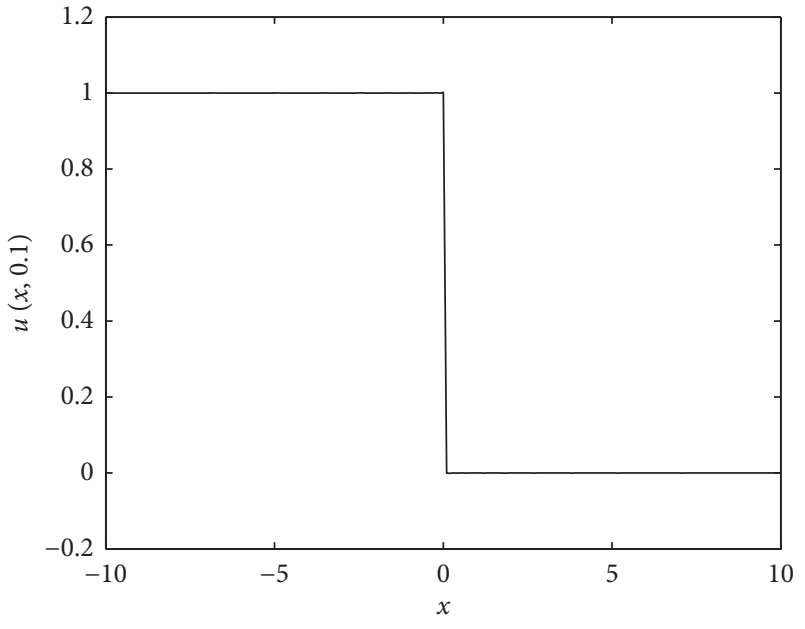

$-\Delta t=0.3 \mathrm{~h}$

(b)

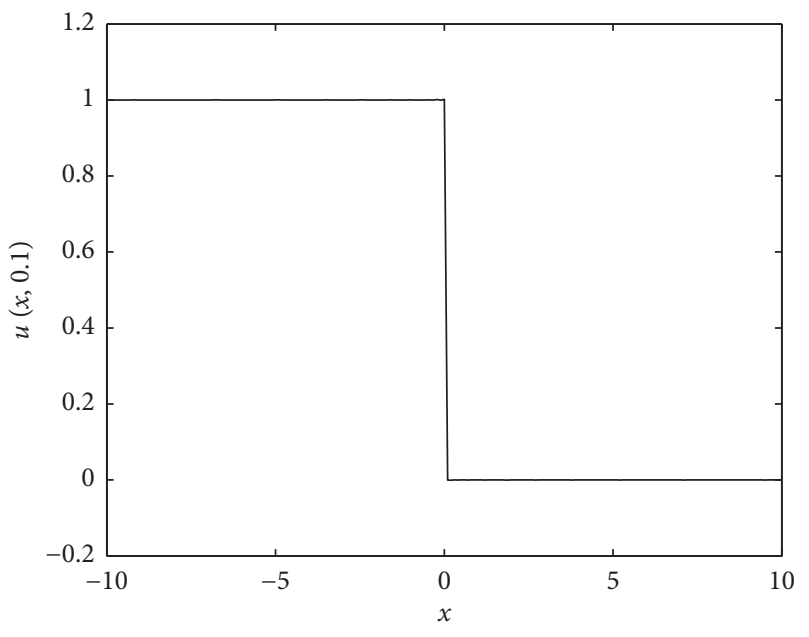

$-\Delta t=0.7 \mathrm{~h}$

(c)

(d)

Figure 5: The numerical solutions of Example 3 with $\alpha=0.9$ and $N=200$ and for different CFL numbers at $T=0.1$. (a) $\sigma=0.1$, (b) $\sigma=0.3$, (c) $\sigma=0.5$, and (d) $\sigma=0.7$. 


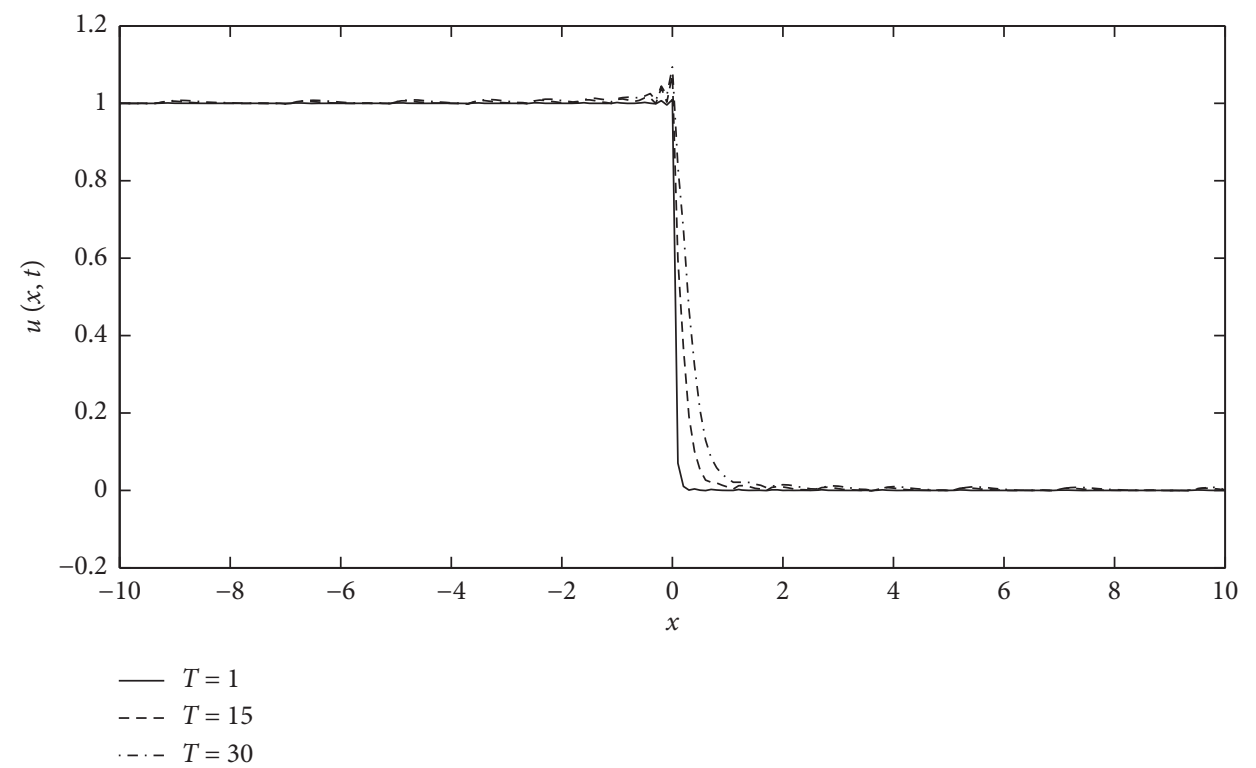

Figure 6: The numerical solutions of Example 3 with $\alpha=0.9$ and $N=200$ at $T=1, T=15$, and $T=30$.

Figure 6 shows the numerical solutions when the parameters are chosen as $\alpha=0.9$ and $N=200$, at $T=1, T=$ 15 , and $T=30$ separately.

\section{Conclusion}

This paper develops the generalized WENO5 methods for numerically solving the spatial fractional neutron transport equation with smooth solutions or piecewise smooth solutions, which is a new attempt in the work of numerical solution for fractional differential equation. By using the classical WENO5 schemes and the Gauss-Jacobi quadrature, we perform the spatial discretization. Then, we get the nonlinear ODE systems, which are computed by the SSP $(3,3)$ Runge-Kutta method and some other Runge-Kutta method. The linear stabilities of the numerical schemes are proved. And, the numerical results are present to verify the effectiveness and reliability of the numerical schemes.

Generally speaking, the WENO scheme is widely used to solve the problem that the solution of the equation is not very smooth and can maintain a high-order convergence rate as well. Therefore, for the upcoming work, we will mainly discuss the discontinuous problems and the nonlinear problems with the WENO method in the future. Maybe, by using the nonuniform mesh method or meshless method together with the WENO scheme for the fractional problems is a good choice.

\section{Data Availability}

No data were used to support this study.

\section{Conflicts of Interest}

The authors declare that they have no conflicts of interest.

\section{Authors' Contributions}

All authors have equally contributed to the article and read and approved the final version of the paper.

\section{Acknowledgments}

This work was supported by the Natural Science Foundation of Shanghai (no. 19ZR1422000), Natural Science Foundation of Anhui Province (no. 2008085MA11), and Natural Science Foundation of Education Department of Anhui Province (no. KJ2018A0385).

\section{References}

[1] A. A. Kilbas, H. M. Srivastava, and J. J. Trujillo, Theory and Applications of Fractional Differential Equations, Elsevier, Amsterdam, Netherlands, 2006.

[2] M. M. Meerschaert and C. Tadjeran, "Finite difference approximations for fractional advection-dispersion flow equations," Journal of Computational and Applied Mathematics, vol. 172, no. 1, pp. 65-77, 2004.

[3] W. Deng, S. Du, and Y. Wu, "High order finite difference WENO schemes for fractional differential equations," Applied Mathematics Letters, vol. 26, no. 3, pp. 362-366, 2013.

[4] H. Sun, Z.-Z. Sun, and G.-H. Gao, "Some temporal second order difference schemes for fractional wave equations," Numerical Methods for Partial Differential Equations, vol. 32, no. 3, pp. 970-1001, 2016.

[5] Z. Hao and W. Cao, "An improved algorithm based on finite difference schemes for fractional boundary value problems with nonsmooth solution," Journal of Scientific Computing, vol. 73, no. 1, pp. 395-415, 2017.

[6] W. H. Deng, "Finite element method for the space and time fractional Fokker-Planck equation," SIAM Journal on $\mathrm{Nu}$ merical Analysis, vol. 47, pp. 204-226, 2008.

[7] V. J. Ervin, N. Heuer, and J. P. Roop, "Numerical approximation of a time dependent, nonlinear, space-fractional 
diffusion equation," SIAM Journal on Numerical Analysis, vol. 45, no. 2, pp. 572-591, 2007.

[8] C. Li, Z. Zhao, and Y. Chen, "Numerical approximation of nonlinear fractional differential equations with subdiffusion and superdiffusion," Computers \& Mathematics with Applications, vol. 62, no. 3, pp. 855-875, 2011.

[9] W. Bu, Y. Tang, and J. Yang, "Galerkin finite element method for two-dimensional Riesz space fractional diffusion equations," Journal of Computational Physics, vol. 276, pp. 26-38, 2014.

[10] B. Jin, R. Lazarov, J. Pasciak, and W. Rundell, "Variational formulation of problems involving fractional order differential operators," Mathematics of Computation, vol. 84, no. 296, pp. 2665-2700, 2015.

[11] Z. Zhang, W. Deng, and G. E. Karniadakis, "A Riesz basis Galerkin method for the tempered fractional Laplacian," SIAM Journal on Numerical Analysis, vol. 56, no. 5, pp. 3010-3039, 2018.

[12] Z. Zhao, Y. Zheng, and P. Guo, "A Galerkin finite element scheme for time-space fractional diffusion equation," International Journal of Computer Mathematics, vol. 93, no. 7, pp. 1212-1225, 2015.

[13] Z. Zhao, Y. Zheng, and P. Guo, "A galerkin finite element method for a class of time-space fractional differential equation with nonsmooth data," Journal of Scientific Computing, vol. 70, no. 1, pp. 386-406, 2017.

[14] Q. Xu and J. S. Hesthaven, "Discontinuous Galerkin method for fractional convection-diffusion equations," SIAM Journal on Numerical Analysis, vol. 52, no. 1, pp. 405-423, 2014.

[15] X. Tian and Q. Du, "Nonconforming discontinuous Galerkin methods for nonlocal variational problems," SIAM Journal on Numerical Analysis, vol. 53, no. 2, pp. 762-781, 2015.

[16] Y. Du, Y. Liu, H. Li, Z. Fang, and S. He, "Local discontinuous Galerkin method for a nonlinear time-fractional fourth-order partial differential equation," Journal of Computational Physics, vol. 344, pp. 108-126, 2017.

[17] Y. Zheng and Z. Zhao, "The discontinuous Galerkin finite element method for fractional cable equation," Applied $\mathrm{Nu}$ merical Mathematics, vol. 115, pp. 32-41, 2017.

[18] C. Li and Z. Wang, "The local discontinuous Galerkin finite element methods for Caputo-type partial differential equations: numerical analysis," Applied Numerical Mathematics, vol. 140, pp. 1-22, 2019.

[19] Y. Zheng, Z. Zhao, and Y. Cui, “The discontinuous Galerkin finite element approximation of the multi-order fractional initial problems," Applied Mathematics and Computation, vol. 348, pp. 257-269, 2019.

[20] Y. Zheng and Z. Zhao, "The time discontinuous space-time finite element method for fractional diffusion-wave equation," Applied Numerical Mathematics, vol. 150, pp. 105-116, 2020.

[21] X. J. Li and C. J. Xu, "Existence and uniqueness of the weak solution of the space-time fractional diffusion equation and a spectral method approximation," Communications in Computational Physics, vol. 8, pp. 1016-1051, 2010.

[22] F. Zeng, F. Liu, C. Li, K. Burrage, I. Turner, and V. Anh, "A crank--nicolson ADI spectral method for a two-dimensional Riesz space fractional nonlinear reaction-diffusion equation," SIAM Journal on Numerical Analysis, vol. 52, no. 6, pp. 2599-2622, 2014.

[23] Z. Mao and G. E. Karniadakis, "A spectral method (of exponential convergence) for singular solutions of the diffusion equation with general two-sided fractional derivative," SIAM Journal on Numerical Analysis, vol. 56, no. 1, pp. 24-49, 2018.
[24] N. Wang, Z. Mao, C. Huang, and G. E. Karniadakis, "A spectral penalty method for two-sided fractional differential equations with general boundary conditions," SIAM Journal on Scientific Computing, vol. 41, no. 3, pp. A1840-A1866, 2019.

[25] H. Hejazi, T. Moroney, and F. Liu, "Stability and convergence of a finite volume method for the space fractional advectiondispersion equation," Journal of Computational and Applied Mathematics, vol. 255, pp. 684-697, 2014.

[26] A. Simmons, Q. Yang, and T. Moroney, "A finite volume method for two-sided fractional diffusion equations on nonuniform meshes," Journal of Computational Physics, vol. 335, pp. 747-759, 2017.

[27] R. Klages, G. Radons, and I. M. Sokolov, Anomalous Transport, Wiley-VCH Verlag GmbH \& Co., New York, NY, USA, 2008.

[28] S. Das and B. B. Biswas, "Fractional divergence for neutron flux profile in nuclear reactors," International Journal of Nuclear Energy Science and Technology, vol. 3, no. 2, pp. 139-159, 2007.

[29] V. A. Vyawahare, P. S. V. Nataraj, G. Espinosa-Paredes, and R.-I. Cázares-Ramírez, "Nuclear reactor with subdiffusive neutron transport: development of linear fractional-order models," International Journal of Dynamics and Control, vol. 5, no. 4, pp. 1182-1200, 2017.

[30] V. A. Vyawahare and P. S. V. Nataraj, "Fractional-order modeling of neutron transport in a nuclear reactor," Applied Mathematical Modelling, vol. 37, no. 23, pp. 9747-9767, 2013.

[31] C. W. Shu, "Efficient implementation of essentially non-oscillatory shock-captring schemes," Journal of Computational Physics, vol. 77, pp. 439-471, 1998.

[32] X.-D. Liu, S. Osher, and T. Chan, "Weighted essentially nonoscillatory schemes," Journal of Computational Physics, vol. 115, no. 1, pp. 200-212, 1994.

[33] G.-S. Jiang and C.-W. Shu, "Efficient implementation of weighted ENO schemes," Journal of Computational Physics, vol. 126, no. 1, pp. 202-228, 1996.

[34] C.-W. Shu, "High order weighted essentially nonoscillatory schemes for convection dominated problems," SIAM Review, vol. 51, no. 1, pp. 82-126, 2009.

[35] R. J. Spiteri and S. J. Ruuth, "A new class of optimal high-order strong-stability-preserving time discretization methods," SIAM Journal on Numerical Analysis, vol. 40, no. 2, pp. 469-491, 2002.

[36] I. Podlubny, Fractional Differential Equations, Academic Press, San Diego, CA, USA, 1999.

[37] R. Wang and R. J. Spiteri, "Linear instability of the fifth-order WENO method," SIAM Journal on Numerical Analysis, vol. 45, no. 5, pp. 1871-1901, 2007.

[38] E. Tadmor, "Stability analysis of finite difference, pseudospectral and Fourier-Galerkin approximations for time-dependent problems," SIAM Review, vol. 29, pp. 469-491, 1987.

[39] D. Levy and E. Tadmor, "From semidiscrete to fully discrete: stability of runge--kutta schemes by the energy method," SIAM Review, vol. 40, no. 1, pp. 40-73, 1998. 